山्山FARANÇAISE

$\gg \mathrm{DE}$

핌AGGGIE

\section{Revue française de pédagogie}

Recherches en éducation

$201 \mid 2017$

Recherche, politique et pratiques en éducation :

services rendus et questions posées d'un univers à

l'autre /2

\title{
WINEBURG Sam. Why Learn History (When It's Already on Your Phone)
}

Chicago/Londres : The University of Chicago Press, 2018, 240 p.

\section{Lucie Gomes}

\section{CpenEdition}

Édition électronique

URL : https://journals.openedition.org/rfp/7346

DOI : 10.4000/rfp.7346

ISSN : 2105-2913

Éditeur

ENS Éditions

\section{Édition imprimée}

Date de publication : 31 décembre 2017

Pagination : 124-126

ISBN : 979-10-362-0148-6

ISSN : 0556-7807

Référence électronique

Lucie Gomes, «WINEBURG Sam. Why Learn History (When It's Already on Your Phone) », Revue française de pédagogie [En ligne], 201 | 2017, mis en ligne le 31 décembre 2017, consulté le 05 janvier 2023. URL : http://journals.openedition.org/rfp/7346 ; DOI : https://doi.org/10.4000/rfp.7346

Ce document a été généré automatiquement le 5 janvier 2023.

Tous droits réservés 


\section{WINEBURG Sam. Why Learn History (When It's Already on Your Phone)}

Chicago/Londres : The University of Chicago Press, 2018, 240 p.

\section{Lucie Gomes}

\section{RÉFÉRENCE}

WINEBURG Sam. Why Learn History (When It's Already on Your Phone). Chicago/Londres :

The University of Chicago Press, 2018, 240 p.

1 Le titre de l'ouvrage de Sam Wineburg est intrigant au premier abord. À quoi bon enseigner l'histoire aujourd'hui puisque tout se trouve dans le téléphone de chacun? Loin d'être un ouvrage pessimiste sur les nouveaux usages des technologies, l'auteur compile plusieurs de ses publications pour réfléchir aux enjeux de l'enseignement de l'histoire dans un monde dans lequel l'accès à l'information pourrait laisser croire qu'il est un accès direct au passé.

2 Sam Wineburg est professeur à l'Université de Stanford où il s'est spécialisé dans la didactique de l'histoire et plus particulièrement dans les recherches sur la pensée historienne. Disposant de fonds conséquents pour ses travaux, il mène des études à la fois sur les élèves, les enseignants mais aussi les historiens pour comprendre ce qui caractérise l'analyse historienne et comment on peut imaginer la mettre en place à l'école. Ainsi il théorise cet " historical thinking » à partir d'études empiriques variées. Il est sans doute le chercheur le plus influent en Amérique du Nord et au-delà dans ce domaine de recherche récent.

3 Ce nouvel ouvrage - qui fait écho à celui de 2001, abondamment cité depuis - est constitué de quatre parties aux titres aussi provocateurs que celui de l'ouvrage luimême: "Notre situation difficile», «La pensée historienne $\neq$ une mémoire plus attractive ", "Penser historiquement à l'ère digitale » et "L'espérance historienne ». Il multiplie au long de son texte les allers-retours entre l'époque de sa jeunesse et le monde d'aujourd'hui pour explorer ce qui a pu changer et comment l'enseignement 
doit s'adapter à ces changements. Ainsi, dès l'introduction, il se remémore le premier exposé qu'il eut à faire, à l'âge de onze ans, et ce bus qu'il fallut prendre pour aller à la bibliothèque si imposante de New York (p. 2). Aujourd'hui, le bus n'est plus nécessaire et l'accès à l'information est devenu plus facile mais, s'appuyant sur une citation de Thomas Jefferson, il pointe le problème que soulève cet accès illimité : la difficile distinction entre la démagogie et la démocratie, et le développement de la faculté des citoyens à être critiques (p. 8).

4 La première partie est un état des lieux consacré à l'attractivité de l'histoire et aux connaissances des élèves sur cette discipline. Tout d'abord, il constate des difficultés de notre temps qu'il pointe, comme le diagnostic récurrent de méconnaissances sur le passé de la part des nouvelles générations. Il part de ce constat fréquent dans les médias et reprend les études menées sur les écoliers depuis plus d'un siècle. Celles-ci sont essentiellement composées de tests à choix multiples dont l'objectif est de ranger les élèves selon une courbe, sortant du lot les meilleurs d'entre eux. Ainsi, il montre que les statistiques de réussites à ces examens de connaissances des élèves ne sont, d'une part, pas pire que par le passé, mais également non représentatives de la faiblesse $\mathrm{du}$ niveau de ceux qui sont interrogés puisque les tests sont conçus pour ne pas être complètement réussis par la grande majorité des élèves (p.25). Peut-on alors considérer que les jeunes d'aujourd'hui connaissent moins le passé que ceux des précédentes générations? Il s'intéresse aussi, dans cette partie, à l'histoire à la mode auprès de la population et auprès des enseignants en se basant sur le succès de l'ouvrage Une histoire populaire des États-Unis d'Howard Zinn. L'intérêt de cet ouvrage, selon Wineburg, est de mettre en valeur l'histoire des minorités mais aussi d'aborder des thèmes sous un angle inhabituel. Son succès pourrait montrer que le renouvellement historiographique rend l'histoire plus intéressante. Cependant, il pointe les défauts de l'ouvrage : il est dépourvu de notes contextuelles, il ne cite pas ses références, et ses sources sont parfois très critiquables d'un point de vue scientifique. La lecture de cet ouvrage par les enseignants ne fait donc que remplacer un récit national standardisé par un autre et n'induit pas en elle-même l'intérêt pour le développement d'une pensée historienne : les lecteurs de l'ouvrage n'ont d'autres choix que de prendre pour argent comptant ce qu'on leur raconte (p. 71).

5 Dans la seconde partie, l'auteur reprend pour les développer les thèses plus directement liées aux relations entre enseignement et apprentissage de l'histoire qu'il a développées dans son ouvrage de référence Historical Thinking (2001). Il compare des étudiants et des historiens sur leurs manières d'aborder une source historique nouvelle pour eux. Les deux groupes sont soumis à la même source et doivent à voix haute exprimer le cheminement de leur analyse. Les historiens en question ne sont pas spécialistes de la période étudiée. Wineburg montre que les étudiants ont davantage de connaissances factuelles que les historiens, car il s'agit d'une période qu'ils ont vu dans leur scolarité. En revanche, la manière de questionner le document diffère de façon conséquente, ce qui permet à Sam Wineburg de poursuivre son travail de caractérisation de la pensée historienne qu'il a entamé dès la fin des années 1980. Il indique que ce qui distingue les historiens réside dans leur autonomie par rapport à la source : ils ne cherchent pas seulement à y collecter des faits de manière critique mais ils se posent une série de questions pour interpréter la source. Ils terminent donc leur lecture de la source avec la volonté d'en savoir plus alors que les étudiants finissent par un jugement à partir de ce qu'ils savent (p. 100). 
6 Dans la troisième partie, il se demande ce que pourrait être un enseignement scolaire de la pensée historienne à l'ère digitale. Ce concept, qu'il a largement contribué à développer par le passé, n'est pas aisé à définir. Connaître des dates, des noms de grands personnages et des batailles est-il caractéristique de cette pensée historienne? Ainsi souhaite-t-il montrer la différence entre des faits non discutables et des faits issus de l'interprétation des historiens. Il prend l'exemple classique aux États-Unis de Rosa Parks : le siège où elle était assise le $1^{\mathrm{er}}$ décembre 1955 , la date de l'évènement, ne sont pas des éléments discutables. En revanche, se poser la question de savoir pourquoi elle a décidé de refuser de céder sa place à un homme blanc ce jour-là relève d'une hypothèse historienne (p.109). C'est pour lui le cœur de la pensée historienne: identifier ce qui dans le passé pose question afin de réfléchir sur les interprétations possibles du passé. C'est donc l'enquête menée sur le passé qui conditionne un mode de pensée historien. À ses contradicteurs qui lui rétorquent que de jeunes élèves n'en sont pas capables, il explique que le problème ne vient pas des écoliers mais des professeurs qui ne savent pas comment enseigner de cette façon.

7 Il se demande ensuite, et cela l'emmène nettement au-delà de ses études antérieures, si l'accès très aisé à des informations très nombreuses, qui peut conduire à tomber dans le piège des fake news et des argumentations complotistes, passe seulement par la nécessité d'apprendre à penser comme les historiens. Pour cela, avec son groupe de recherche de Stanford, il a soumis des historiens et des non-historiens à une série de sites dont ils devaient valider la fiabilité. Le postulat de départ était que la pensée historienne est un atout pour démêler les sources sur Internet. Les résultats de son étude sont surprenants: les historiens ne sont pas plus habiles que les nonscientifiques pour juger de la fiabilité des informations disponibles en ligne. Il explore les raisons de ce constat en comparant la façon dont les historiens et les «factcheckers» lisent une page Internet. Ces derniers sont des spécialistes pour débusquer les informations falsifiées sur Internet. Souvent journalistes eux-mêmes, ils ont dû s'adapter aux nouveaux médias. Il montre que la différence méthodologique entre eux et les historiens réside dans la façon de parcourir les sites auxquels ils sont soumis et de chercher ailleurs sur Internet. Ainsi, les historiens ont une lecture verticale qui les induit en erreur. À l'inverse, les "fact-checkers» ont une lecture horizontale qui consiste à ouvrir de multiples fenêtres en même temps sur leurs ordinateurs pour comparer et mener l'enquête entre sources fiables et sites peu crédibles (p. 152).

Dans la dernière partie, Sam Wineburg essaie de redonner de l'espoir à l'enseignement de l'histoire aujourd'hui, en en reconsidérant les finalités. Si les élèves ont en effet accès à une somme considérable d'informations, ils n'ont en revanche pas le mode d'emploi pour les trier et les sélectionner afin de réfléchir sur le passé. Il conseille donc d'arrêter de confisquer les téléphones à l'école et de plutôt apprendre aux élèves à s'en servir dans une nouvelle forme de critique historienne, tout en leur montrant ce que leur téléphone ne peut pas faire. Il reste donc à explorer des pistes didactiques pour atteindre cet objectif.

9 En plein débat sur l'usage du téléphone portable à l'école, son ouvrage peut aider à repenser le débat sur les instruments et les usages des réseaux sociaux et autres sites d'information. Il ne s'agit pas pour lui de se passer des livres et des documents classiques. Mais que valent ces supports face à l'attractivité d'un écran allumé ? Prendre en compte le fait que déambuler dans les méandres d'Internet nécessite un apprentissage autre que la pensée historienne traditionnelle est un apport conséquent 
de son ouvrage. Si même des historiens peinent à identifier les indices de fiabilité d'un site, c'est qu'on ne peut imaginer que développer chez les élèves la pensée historienne les rend automatiquement compétents avec le numérique. Il reste donc à inventer ces nouvelles formes d'apprentissages.

\section{AUTEURS}

\section{LUCIE GOMES}

Université de Nantes, CREN 